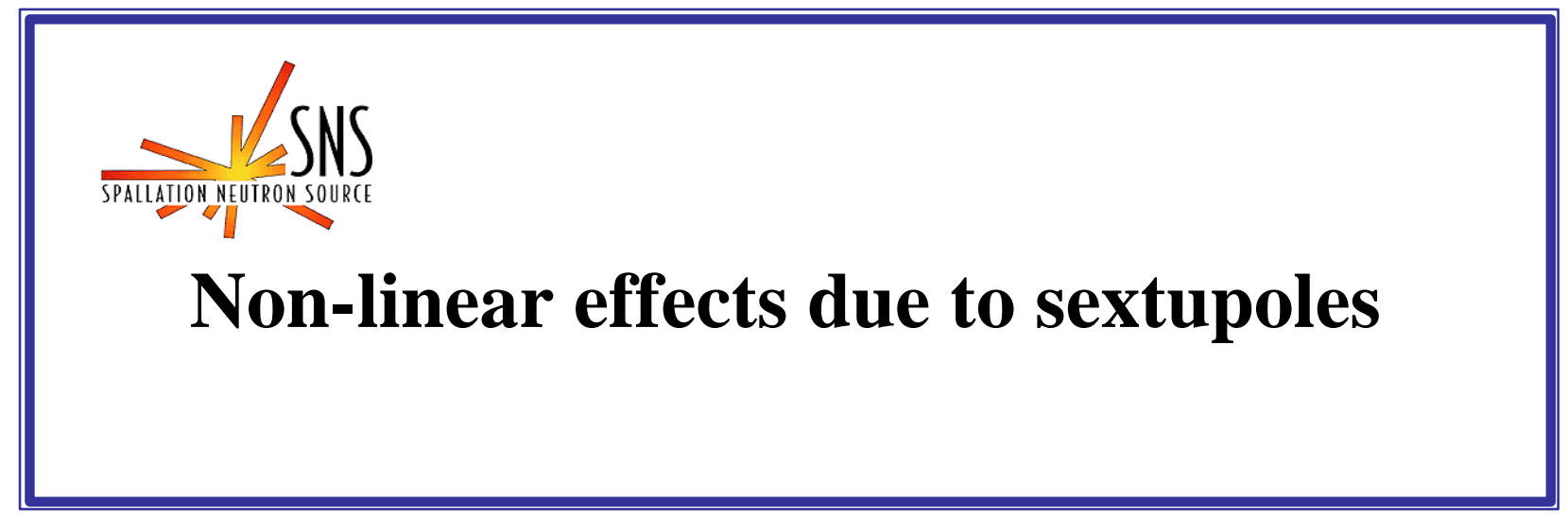

BNL/SNS TECHNICAL NOTE

NO. 105

(REVISED)

G. Parzen

March 20, 2002

COLLIDER-ACCELERATOR DEPARTMENT BROOKHAVEN NATIONAL LABORATORY UPTON, NEW YORK 11973 


\title{
Non-linear effects due to sextupoles
}

\author{
George Parzen
}

March 20, 2002

\section{Non-linear effects due to sextupoles}

The sextupoles used to correct the chromaticity introduce non-linear fields into the ring. The non-linear effects due to these sextupoles may be studied by computing the dynamic aperture [1] and by computing the tune as a function of the betatron oscillation amplitude[2]. These studies indicate that the non-linear effects due to these sextupoles are small. Another approach to estimating the non-linear effects is to compute the emittance growth due to the sextupoles. This approach is used in this note and shows a small but appreciable non-linear effect due to the sextupoles.

Consider a group of particles where each of these particles are started having the same linear emittances, $\epsilon_{x}, \epsilon_{y}$ but with different starting values of $x, p_{x}, y, p_{y}$. In the absence of the sextupoles, these particles will reach a maximun value of $x$, denoted by $x_{\max }$, and a maximun value of $y, y_{\max }$, at any place around the ring, which can be computed using $\epsilon_{x}, \epsilon_{y}$ and the beta functions, $\beta_{x}, \beta_{y}$ at that place. When the sextupoles are present, some particles in this group will reach values of $y_{\max }$ or $x_{\max }$ which are larger than than those allowed by the linear motion in the absence of sextupoles. In this sense, one may say that the sextupoles cause some growth in the beam size. This growth in the beam size may be decribed by the results of an emittance growth study in which the above group of particles is tracked through a given number of turns and the maximun values of $\epsilon_{x}, \epsilon_{y}$ and $\epsilon_{t}=\epsilon_{x}+\epsilon_{y}$ reached by all the particles in the group are recorded as $\epsilon_{x \max }, \epsilon_{y \max }, \epsilon_{\text {tmax }}$. The emittances are computed using the $\beta_{x}, \alpha_{x}, \beta_{y}, \alpha_{y}$ of the linear motion in the absence of sextupoles. The study could also compute $x_{\max }, y_{\max }$ of this group of particles at some places in the ring. It is sometimes more convenient

to compute $x_{\max }, y_{\max }$ from $x_{\max }=\left(\beta_{x} \epsilon_{x \max }\right)^{.5}, y_{\max }=\left(\beta_{y} \epsilon_{y \max }\right)^{.5}$, which is 
valid if the non-linear effects due to the sextupoles are not too strong. One should note that this method of computing $x_{\max }, y_{\max }$ is not exact. For the SNS ring it overestimates $x_{\max }, y_{\max }$ by about $5 \%$.

The method of the emittance growth study can be applied to the SNS ring. The results of an emittance growth study, more carefully defined below, give $\epsilon_{x \max }=144, \epsilon_{y \max }=155, \epsilon_{t \max }=280$ for a group of particles each of which was started with $\epsilon_{x}=\epsilon_{y}=120$, which is at the outer boundary of the beam. All emittances are in mm. mrad. The emittance growth of $\epsilon_{y}$ from 120 to 155 indicates a growth in vertical beam size of about $8 \%$. One may note that the growth in $\epsilon_{y}$ is about $29 \%$, but the corresponding growth in vertical beam size is about $8 \%$, which is smaller than the result found from $y_{\max }=\left(\beta_{y} \epsilon_{y \max }\right)^{.5}$. The significance of the growth of $\epsilon_{t}$ from 240 to 280 is not clear. It depends on what other mechanism is present, besides the sextupoles, that can couple the transverse motions.

\section{Computed results for the emittance growth}

In Fig. 1, a group of particles with $\Delta p / p=0$ are tracked for 100 turns when two families of sextupoles are set to make the chromaticity equal to zero, $C_{x}=0, C_{y}=0$ using the lattice with the tune $\nu_{x}=6.23, \nu_{y}=6.20$. The emittances, $\epsilon_{x}, \epsilon_{y}$ are plotted at the beginning of each element of the lattice for each of the partcles in the group. The particles in the group are all started with $\epsilon_{x}=\epsilon_{y}=120$ mm.mrad., and $\epsilon_{t}=\epsilon_{x}+\epsilon_{y}=240 \mathrm{~mm}$. mrad., but with different $x, p_{x}, y, p_{y}$. If the sextupoles were not present, the plot would be a single point at $\epsilon_{x}=\epsilon_{y}=120$. When the sextupoles are present, the emittances are no longer constant. $\epsilon_{x}, \epsilon_{y}, \epsilon_{t}$ reach maximun values of $\epsilon_{x}=144, \epsilon_{y}=155, \epsilon_{t}=280$. This growth in the emitances happens in less than 10 to 20 turns. The growth in $\epsilon_{y}$ from 120 to $155 \mathrm{~mm}$. mrad.is about $29 \%$. The corresponding growth in $y_{\max }$ was found to be about $8 \%$ which is smaller than the result indicated by $y_{\max }=\left(\beta_{y} \epsilon_{y \max }\right)^{.5}$ by $6 \%$.

Most of the computations in this study were done with a group of 4 particles tracked for 100 turns. The group contained 4 particles each starting with $\epsilon_{x}=\epsilon_{y}=120 \mathrm{~mm}$.mrad.but with different starting $x, p_{x}, y, p_{y}$. Running with more particles or running for more turns does not change the results by much.

The region of $\mathrm{x}, \mathrm{y}$ space that is reached by the group of particles being studied is rectangular in shape when the sextupoles are absent. When 
the sextupoles are present, the shape of the $\mathrm{x}, \mathrm{y}$ space reached is shown in Fig. 2. The shape is shown at the middle of the long straight section where $\beta_{x}=10.43, \beta_{y}=12.11 \mathrm{~m}$. The shape is still roughly rectangular, but the dimensions of the rectangle have been increased from $\pm 35.4 b y \pm 38.1 \mathrm{~mm}$ to $\pm 37.2 b y \pm 40.8 \mathrm{~mm}$.

If the chromaticity sextupoles are not excited, then the ring has its natural chromaticity which is about -7.5 . Thus one expects that as one lowers the chromaticity from 0 toward -7.5 , the strength of the sextupoles will decrease and the emittance growth will also decrease. This is shown in Fig. 3 where the maximun total emittance reached, $\epsilon_{t, \max }$, is plotted against the chromaticity, $C=C_{x}=C_{y} . \epsilon_{t, \max }$ is computed by tracking a group of particles through 100 turns where the particles in the group are all started with $\epsilon_{x}=\epsilon_{y}=120$

mm.mrad., and $\epsilon_{t}=\epsilon_{x}+\epsilon_{y}=240$ mm,mrad., but with different $x, p_{x}, y, p_{y}$. Results are shown for $\Delta p / p=0$.

\section{References}

[1] A. Fedotov, D.T. Abell, .Y Lee. N. Malitsky, Y. Papaphilippou, J.Wei, EPAC 2000, (2000)

[2] Y. Papaphilippou and N. Tsoupas, BNL/SNS Tech. Note No. 075, (2000) 


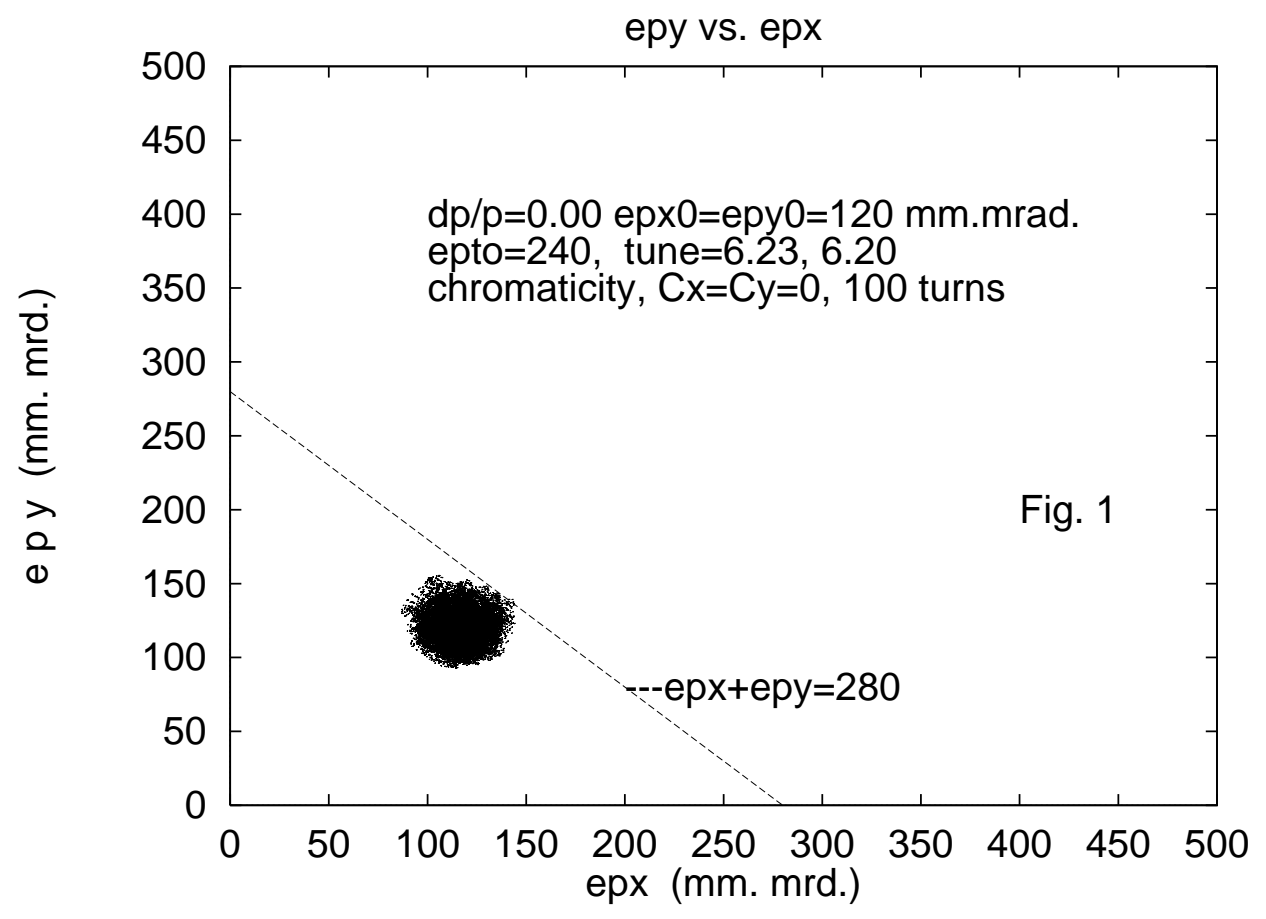

Figure 1: $\epsilon_{y}$ vs. $\epsilon_{x}$ plotted at the beginning of each element of the lattice for each of the partcles in a group of particles tracked for 100 turns, with $\epsilon_{y 0}=\epsilon_{x 0}=120$ mm.mrad., $\epsilon_{t 0}=240$ mm.mrad. but with different initial $x, p_{x}, y, p_{y}$. Chromaticities $C_{x}=C_{y}=0$ and $\Delta p / p=0$. In the figure, epx, epy, ept, epx0, epy0, ept0 represent $\epsilon_{x}, \epsilon_{y}, \epsilon_{t}, \epsilon_{x 0}, \epsilon_{y 0}, \epsilon_{t 0}$ 


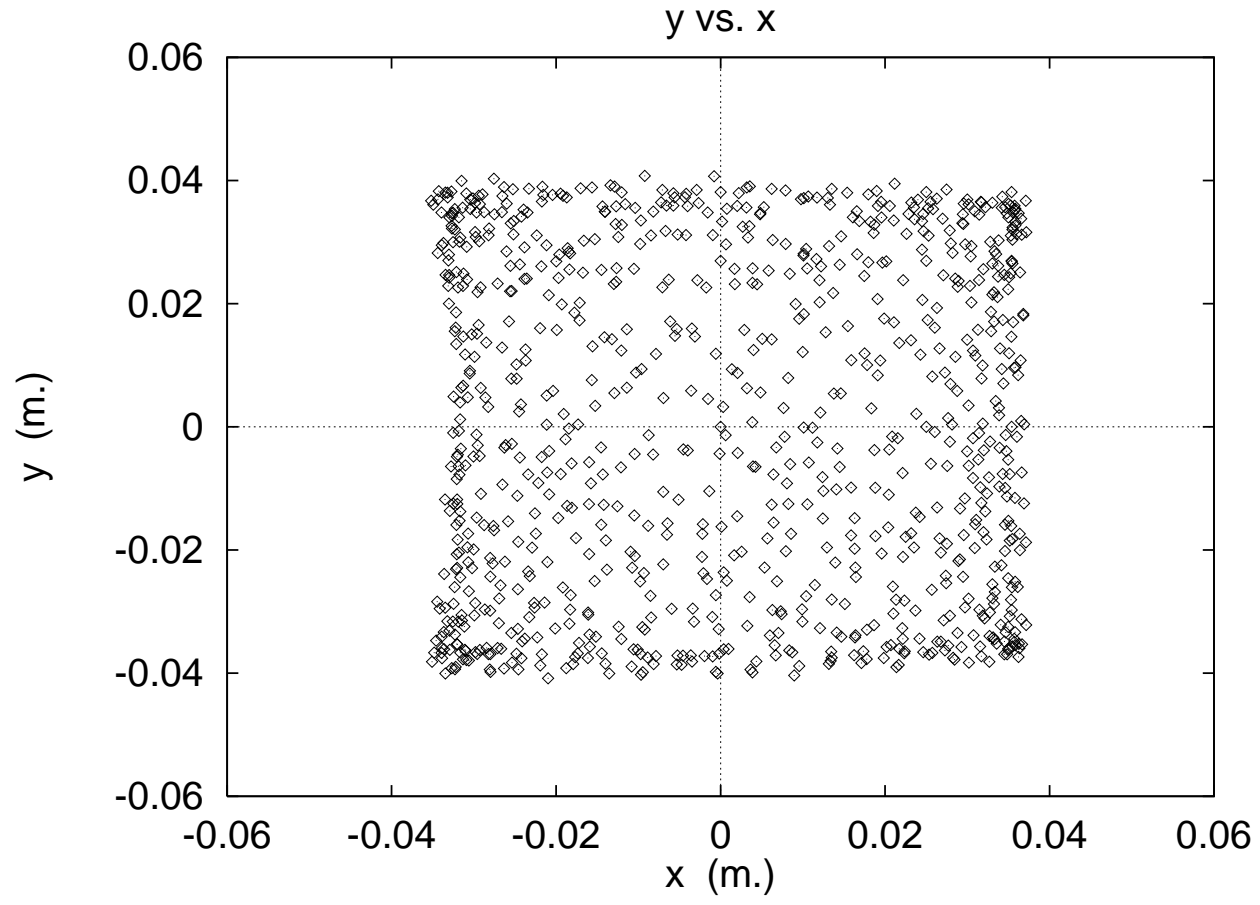

Figure 2: $y$ vs. $x$ plotted at the beginning of each element of the lattice for each of the partcles in a group of particles tracked for 100 turns, with $\epsilon_{y 0}=\epsilon_{x 0}=120$ mm.mrad., $\epsilon_{t 0}=240 \mathrm{~mm} . \mathrm{mrad}$. but with different initial $x, p_{x}, y, p_{y}$. Chromaticities $C_{x}=C_{y}=0$ and $\Delta p / p=0$. 


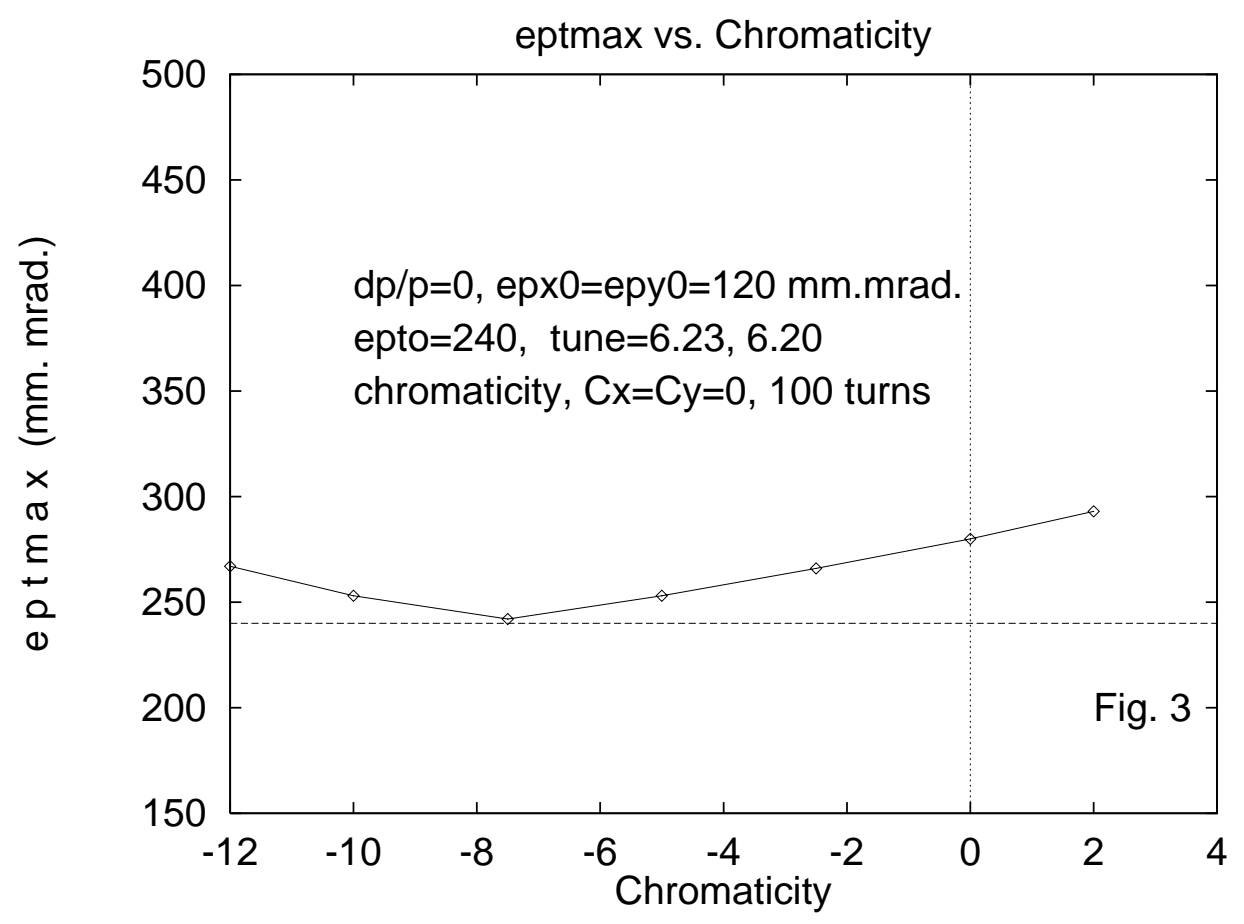

Figure 3: $\epsilon_{t, \max }$ vs. Chromaticity by tracking a group of particles through 100 turns where the particles in the group are all started with $\epsilon_{x}=\epsilon_{y}=120$ mm.mrad., and $\epsilon_{t}=\epsilon_{x}+\epsilon_{y}=240$ mm,mrad., but with different $x, p_{x}, y, p_{y}$. Horizontal and vertical chromaticity are equal and $\Delta p / p=0$.In the figure, epx, epy, ept, epx0, epy0, ept0, eptmax represent $\epsilon_{x}, \epsilon_{y}, \epsilon_{t}, \epsilon_{x 0}, \epsilon_{y 0}, \epsilon_{t 0}, \epsilon_{t, \max }$ 